\title{
The Arabidopsis PRC1-like ring-finger proteins are necessary for repression of embryonic traits during vegetative growth
}

\author{
Donghong Chen ${ }^{1}$, Anne Molitor ${ }^{1}$, Chunlin Liu ${ }^{2}$, Wen-Hui Shen ${ }^{1}$ \\ ${ }^{1}$ Institut de Biologie Moléculaire des Plantes du CNRS, Université de Strasbourg, 12 rue du Général Zimmer, 67084 Strasbourg \\ Cedex, France; ${ }^{2}$ Hunan Provincial Key Laboratory of Crop Germplasm Innovation and Utilization, Hunan Agricultural University, \\ Changsha 410128, China
}

Polycomb group genes play crucial roles in the maintenance of the transcriptionally silenced state of genes for proper cell differentiation in animals and plants. While components of the polycomb repressive complex2 (PRC2) are evolutionarily conserved and their functions are extensively studied in plants, PRC1 differs considerably between animals and plants, and its functions in plants are as yet not well described. Previous studies have identified the Arabidopsis AtRING1a and AtRING1b as homologues of the animal PRC1 subunit RING1. Here, we show that the Atring1a Atring1b double mutant exhibits derepression of embryonic traits during vegetative growth. Accordingly, several key regulatory genes involved in embryogenesis and stem cell activity are ectopically expressed in the mutant. Furthermore, we show that the mutant phenotypes and increased expression of regulatory genes are enhanced by the PRC2 mutant $c l f$. Finally, we show that three homologues of the animal PRC1-subunit ring-finger protein BMI1, AtBMI1a, AtBMI1b and AtBMI1c, can bind with AtRING1a or AtRING1b, and in addition, AtBMI1c can bind with LHP1. The Atbmila Atbmilb double mutant shows derepression of embryonic traits similar to that of the Atring1a Atringlb double mutant. Interestingly, expression levels of AtBMI1a, AtBMI1b and AtBMI1c are elevated in the Atring1a Atring1b mutant and those of AtBMI1c, AtRING1a and AtRING1b are elevated in the Atbmila Atbmilb mutant, suggesting a self-regulatory feedback mechanism. Taken together, our results illuminate crucial functions of the PRC1-like ring-finger components in stable repression of embryonic traits and regulatory genes for proper somatic growth.

Keywords: Polycomb; PRC1; RING1; somatic embryogenesis; Arabidopsis

Cell Research (2010) 20:1332-1344. doi:10.1038/cr.2010.151; published online 9 November 2010

\section{Introduction}

Polycomb group $(\mathrm{PcG})$ proteins exist in multiprotein complexes; the best characterized of these are known as polycomb repressive complex1 (PRC1) and PRC2 in Drosophila and mammals. PRC2, via its catalytic subunit $\mathrm{E}(\mathrm{z})$, methylates histone $\mathrm{H} 3$ lysine 27 (H3K27), resulting in trimethyl-H3K27 (H3K27me3), and PRC1, via its chromodomain-containing subunit polycomb $(\mathrm{Pc})$, binds

Correspondence: Wen-Hui Shen

E-mail: wen-hui.shen@ibmp-cnrs.unistra.fr

Received 11 August 2010; revised 14 September 2010; accepted 12 October 2010; published online 9 November 2010
$\mathrm{H} 3 \mathrm{~K} 27 \mathrm{me} 3$ resulting in a stable silencing chromatin state (reviewed in Schuettengruber et al.[1]). In the model plant Arabidopsis thaliana, PRC2 subunits are evolutionarily conserved, thus MEDEA (MEA), CURLY LEAF (CLF) and SWINGER (SWN) are E(z) homologues; EMBRYONIC FLOWER2 (EMF2), FERTILISATION INDEPENDENT SEED2 (FIS2) and VERNALIZATION2 (VRN2) are $\mathrm{Su}(\mathrm{Z}) 12$ homologues; FERTILIZATION INDEPENDENT ENDOSPERM (FIE) is the unique ESC homologue; and MULTICOPY SUPRESSOR OF IRA1-5 (MSI1-5) are RbAp46/48 homologues, but only MSI1 has been demonstrated as part of the PRC2 complexes so far (reviewed in Pien and Grossniklaus[2] and Alvarez-Venegaz [3]). Homologues of PRC2 complexes are involved in many aspects of plant development, in- 
cluding the repression of flowering during vegetative development, the suppression of endosperm development in the absence of fertilization, and the repression of stem cell pluripotency for cell differentiation (reviewed in Pien and Grossniklaus[2], Alvarez-Venegaz [3] and Shen and $\mathrm{Xu}$ [4]). In comparison, much less is known about PRC1 function in plants. Arabidopsis does not contain any homologue of Pc. Nevertheless, the chromodomaincontaining protein LIKE HETEROCHROMOTIN PROTEIN1 (LHP1) binds H3K27me3 and thus can play a Pcanalogous function in Arabidopsis [5, 6]. In addition to $\mathrm{Pc}$, the animal PRC1 core complex contains ring-finger proteins RING1A, RING1B and BMI1 [1]. Arabidopsis has two RING1-homologues, AtRING1a and AtRING1b, which bind to LHP1 and together are involved in repression of Class I KNOX genes for the maintenance of proper shoot stem cell activity [7]. Arabidopsis also contains three genes encoding ring-finger proteins that show closer homology to BMI1 [7, 8], which we name accordingly hereinafter as AtBMI1a (At1g06770), AtBMI1b (At2g30580) and AtBMI1c (At3g23060). Qin et al. [9] reported that AtBMI1a (also named DRIP1) and AtBMI1b (also named DRIP2) are involved in ubiquitindependent proteasomal degradation of the transcriptional regulator DREB2A, which is involved in drought stress response. The possible PRC1-like function of AtBMI1a, AtBMI1b and AtBMI1c is unexamined to date. In this study, we show that the double mutant Atringla Atringlb, as well as Atbmila Atbmilb, exhibits derepression of embryonic traits in somatic plant tissues, and that AtRING1a and AtRING1b bind AtBMI1a, AtBMI1b and AtBMI1c. We propose that AtRING1 and AtBMI1 proteins have non-redundant functions within a PRC1like complex, which is crucial for the maintenance of differentiated somatic cell fate during post-embryonic plant development.

\section{Results}

AtRING1a::AtRING1a-GUS is expressed at high levels in vegetative tissues containing actively proliferating cells

Previous RT-PCR analysis showed that AtRING1a and AtRING1b are ubiquitously expressed in several examined plant organs including roots, stems and leaves [7]. In order to further investigate AtRING1a expression, we constructed AtRINGla::AtRING1a-GUS, which contains the AtRING1a promoter (-1 $359 \mathrm{bp}$ ) and entire coding region (+4 $356 \mathrm{bp}$; including all introns and exons) fused in frame with the $\beta$-glucuronidase reporter gene GUS. Transgenic plants containing AtRING1a::AtRING1aGUS were investigated for GUS activity by histochemi- cal staining.

While GUS activity was undetectable in imbibed seeds (Figure 1A), GUS staining was clearly visible in the root apical meristem (RAM) as early as 1 day after seed stratification (DAS) (Figure 1B) and in the RAM and shoot apical meristem (SAM) starting from 2 DAS (Figure 1C). This appearance of GUS staining over time correlates relatively well with the previously established time transition from quiescent to active cell proliferation in RAM and SAM during seed germination [10]. At later growth stages, GUS staining was observed in young leaves and vasculature of older leaves (Figure 1D), where cell proliferation is active; in the junction zone between the root and shoot (Figure 1E), where adventitious roots initiate; in lateral root primordium (Figure $1 \mathrm{~F}$ ); and at all stages of growth in RAM (Figure $1 \mathrm{G}$ ) and SAM (Figure 1D). Together, these results indicate that AtRING1a::AtRING1a-GUS expression is closely associated with actively proliferating cells.

The double mutant Atringla Atring1b displays derepression of embryonic traits

Our previous work demonstrated SAM defects in the double mutant Atring la Atring 1b [7]; however, the cell fate of abnormal tissue generation in the mutant had not been investigated. The observation that AtRING1a::AtRING1a-GUS expression is associated with cell proliferation early after the break of seed dormancy prompted us to examine whether AtRING1a and AtRING1b play a role in the maintenance of somatic cell fate in post-embryonic plant development. We investigated the Atringla Atringlb-mutant phenotype of plants grown on in vitro culture medium. We used Fat Red dye staining to reveal the presence of neutral lipids, which accumulate during seed maturation and disappear during vegetative growth, thus serving as an indicator of embryonic traits [11]. As expected, no red staining was detectable in 1-month-old wild-type plants (Figure 2A). Interestingly, the Atringla Atringlb mutant showed red staining of SAM and RAM zones in growth-arrested plants (Figure 2B). Staining was also observed in ectopic calli formed on cotyledons, leaves or from the SAM zone of mutant plants (Figure 2B and 2C). The mutant embryonic calli (EC) proliferated and could produce leaf-like structures (Figure 2D and 2E). The differentiated leaflike structures largely do not display embryonic traits; red staining is visible only on undifferentiated parts of EC (Figure 2D and 2E). In addition to red staining, the distal end of primary root was swollen and greenish in appearance. A similar root phenotype was previously described in the pickle ( $p k l$ ) mutant [12]; thus, we hereinafter use pickle-root to describe the Atringla Atringlb- 

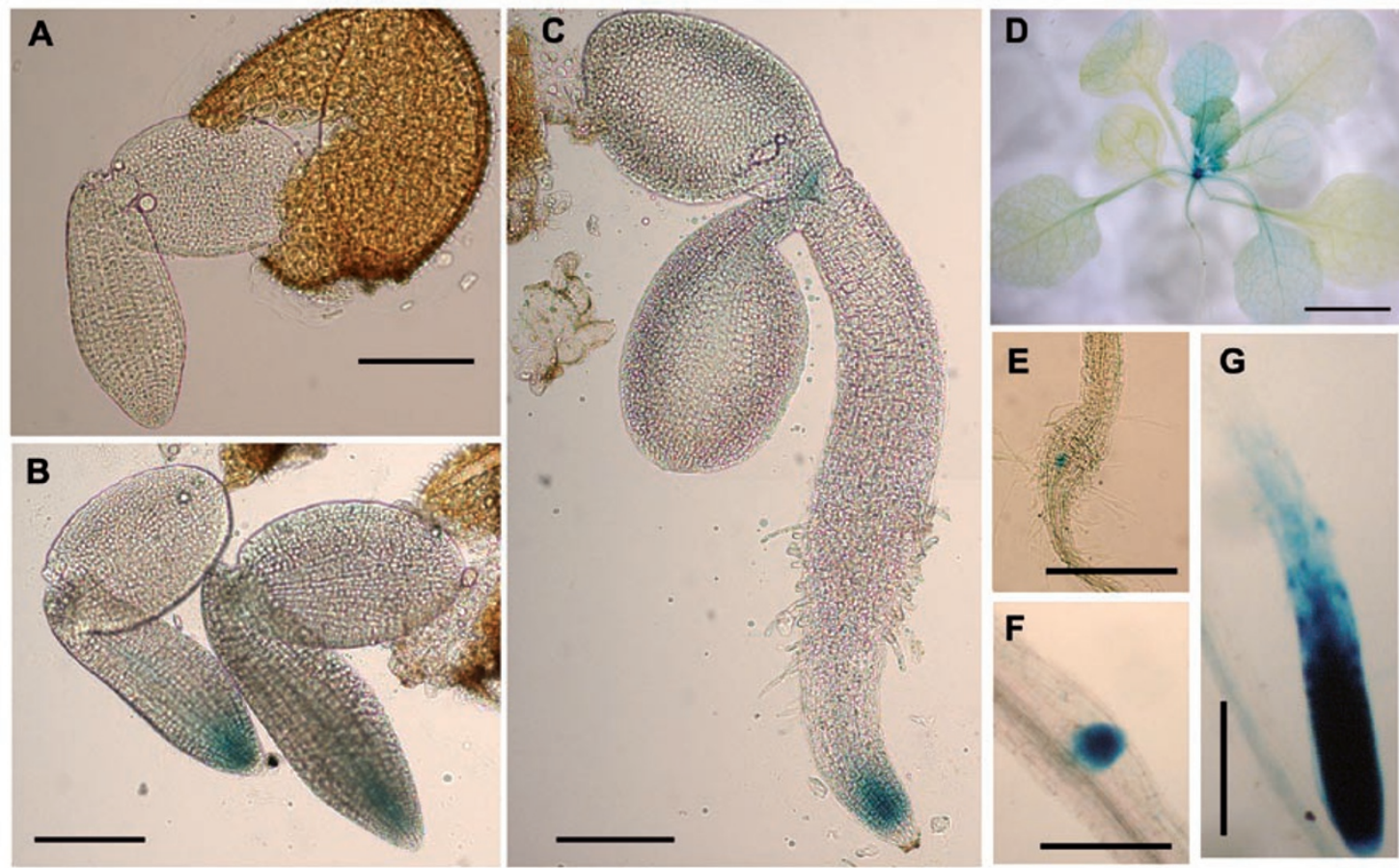

Figure 1 Spatiotemporal expression of AtRING1a::AtRING1a-GUS in transgenic Arabidopsis plants. (A) Imbibed seeds at 4 ${ }^{\circ} \mathrm{C}$ for 2 days. (B) Seedlings grown on culture medium at 1 day after stratification (DAS). (C) Seedling at 2 DAS. (D, E, F, G) Aerial part, shoot-root junction, lateral root primordium region and primary root tip, respectively, from a 1-month-old seedling. Samples were incubated in GUS staining solutions for $2 \mathrm{~h}$ (A-D) or for $16 \mathrm{~h}$ (E-G). Note blue staining that indicates presence of GUS activity. Scale bar = $250 \mu \mathrm{m}$ (A-C), $2 \mathrm{~mm}$ (D) and $100 \mu \mathrm{m}$ (E-G).

mutant root phenotype. Starch accumulation is another seed trait; while only a few columella cells contain starch granules in wild-type roots, the pickle-roots from Atringla Atring $1 b$ showed additional starch granule accumulation at the distal end (Figure 2F), further revealing the abnormal presence of embryonic traits in somatic cells of the Atringla Atringlb mutant. The pickle-root tip is thicker, contains more cell layers, and is arrested in elongation (Figure 2G). The EC and pickle-root phenotypes are not fully co-segregated in the Atringla Atringlb mutant. The penetrance of EC and pickle-root was roughly $17 \%$ and $50 \%$, respectively, and together account for $\sim 58 \%$ of Atringla Atringlb-mutant plants showing derepression of embryonic traits (Figure $2 \mathrm{H}$ ).

AtRING1 $a$ and AtRING1b are required for stable repression of key regulatory genes involved in embryogenesis and stem cell activity

To investigate the molecular events underlying derepression of embryonic traits in Atring la Atringlb-mutant plants, we analyzed expression levels of selected key regulatory genes involved in stem cell activity and embryogenesis (Figure 3A). Consistent with our previous findings [7], the key SAM-regulatory genes (STM, BP/ KNAT1, KNAT2 and KNAT6) encoding Class I KNOX transcription factors are upregulated by 2 - to 6-fold in the mutant. The NAC-domain transcription factor genes $C U C 1, C U C 2$ and $C U C 3$, which are required for organ boundary establishment and SAM initiation [13], are upregulated by 3 - to 15 -fold in the double mutant (Figure $3 \mathrm{~A})$. While the homeodomain transcription factor gene WUS and its homologue WOX2, which are essential for SAM organizing center activity and apical embryo-axis cell fate $[14,15]$, are only slightly upregulated, WOX5 and $W O X 8$, which are crucial for RAM function and basal embryo-axis cell fate termination [15], are upregulated by more than 5 -fold in the mutant (Figure 3A). The embryonic competence-enhanced factor gene $A G L 15$ [16] is upregulated by more than 15-fold, whereas expression of the somatic embryogenesis receptor-like kinase genes SERK1 and SERK2 [17] is unaffected in the mutant (Figure 3A). Drastic upregulation of expression (from 18- to more than 360-fold) was observed for several key embryonic regulatory genes (Figure $3 \mathrm{~A}$ ), including $B B M$ encoding an AP2/ERF transcription factor [18], LEC1 encoding a CCAAT-binding transcription factor [19], as 

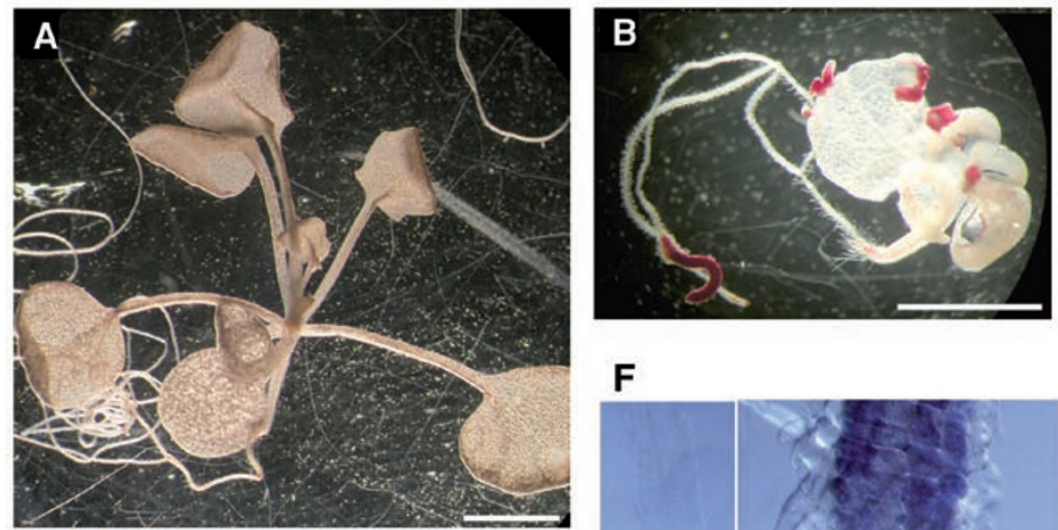

$\mathbf{F}$
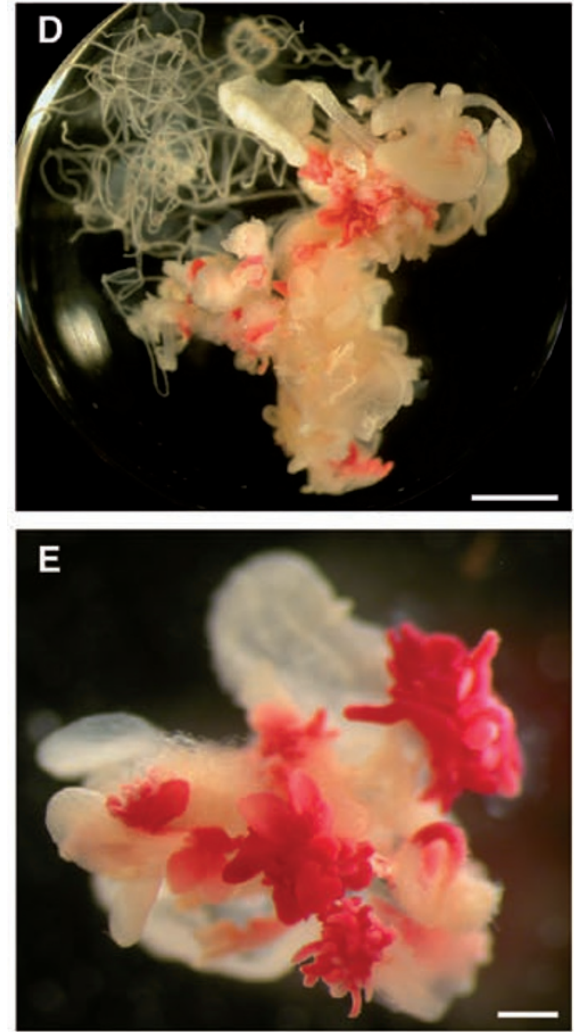

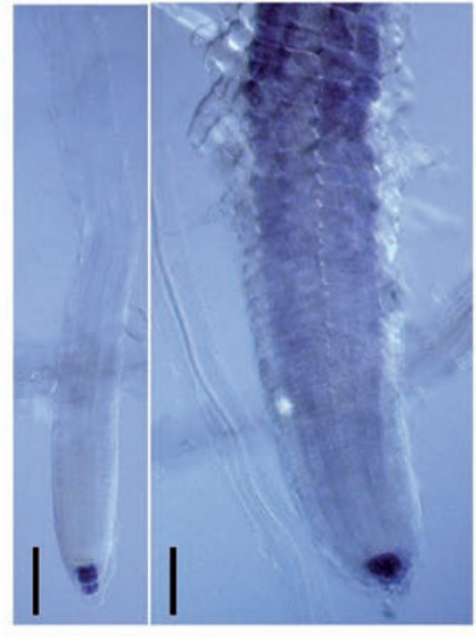

H

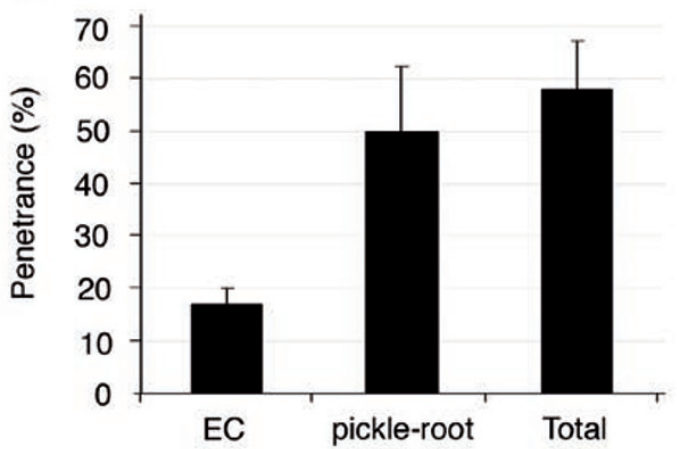

Figure 2 Phenotypes of the Atring1a Atring1b-mutant plants. (A) Wild-type plant (1-month old) stained by Fat Red. (B, C) Atring1a Atring1b plants (1-month old) stained by Fat Red. Note embryonic calli (EC) and pickle-root segments that display triacylglycerol accumulation stained in red. (D, E) Atring1a Atring1b plants (2-month old) stained by Fat Red. Note growth of leaf-like structures from EC. (F) lodo-starch staining of wild-type (left panel) and Atring1a Atring1b (right panel) roots. Note starch granules (in dark color) that appear in columella cells at tip of both wild-type and mutant roots, and in pickle-root cells at distal zone of mutant roots only. (G) Close-up of root tip from wild-type (upper panel) and Atring1a Atring1b (lower panel). (H) Quantitative analysis showing penetrance of EC and pickle-root phenotypes in 1-month-old Atring1a Atring1b plants. Total shows percentage of plants exhibiting EC and/or pickle-root phenotypes. A sum of 578 plants were scored. Error bars represent standard deviation from triplicate repeats. Scale bar $=1 \mathrm{~mm}(\mathbf{A}-\mathbf{C}), 5 \mathrm{~mm}(\mathbf{D}), 1 \mathrm{~mm}(\mathbf{E}), 100 \mu \mathrm{m}(\mathbf{F})$ and $50 \mu \mathrm{m}(\mathbf{G})$.

well as LEC2, FUS3 and ABI3 encoding B3 domain factors [20-22]. It is known that the phytohormone auxin plays an important role in embryogenesis and somatic embryo formation [23]. We detected a 3- to 4-fold up- regulation of PIN1 and PIN2 in the mutant, but neither PIN4 nor PIN7 expression was affected, all from a gene family encoding polar auxin transporters [24] (Figure $3 \mathrm{~A})$. Taken together, our results show that some but not 
all stem cell and embryonic regulatory genes are ectopically derepressed in Atringla Atringlb.

To further investigate the association of gene expression and the mutant phenotype, we introduced by genetic cross the ABI3::GUS [25] and CYCB1::GUS [26] reporter genes into the Atringla Atring $1 b$ mutant. While $A B I 3:: G U S$ expression is restricted to the SAM zone in wild-type plants (left panel in Figure 3B), in the Atringla Atring $1 b$-mutant plants, GUS staining is, in addition, clearly visible in some regions of cotyledons (right panel in Figure 3B) and leaves (data not shown). This ectopic expression pattern of $A B I 3: \because G U S$ is very similar to that of $B P:: G U S$ (Figure 3C; $\mathrm{Xu}$ and Shen [7]), indicating that both genes are ectopically derepressed in somatic cells of the Atring la Atring $1 b$ mutant. The reporter $C Y C B 1: \because G U S$ is expressed during late $\mathrm{G} 2$ and $\mathrm{M}$ phases of the cell cycle, thus providing a marker of cell division [26]. In wild-type seedlings, GUS activity is detected
A

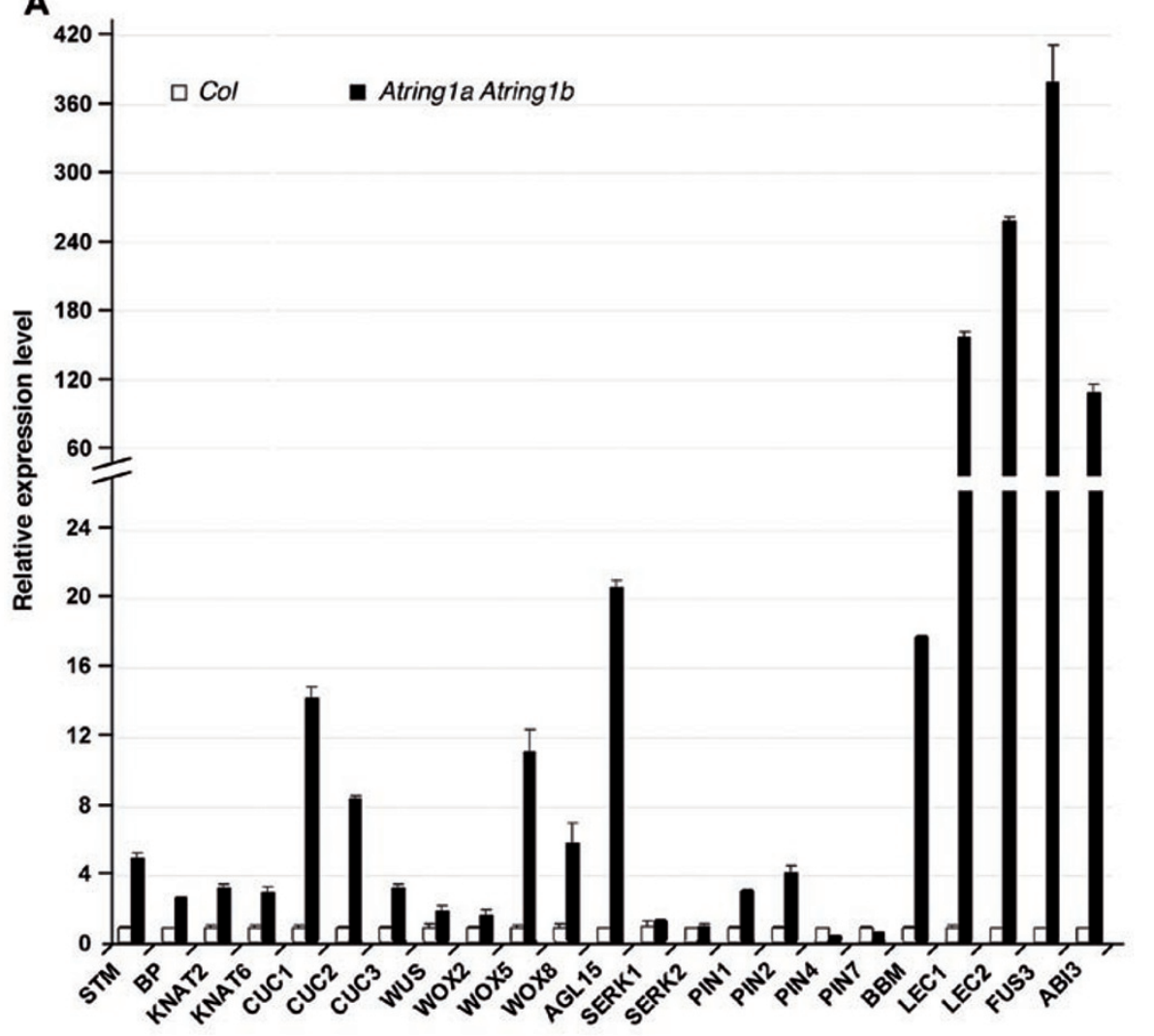

C BP::GUS

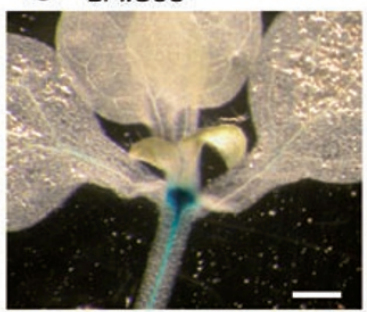

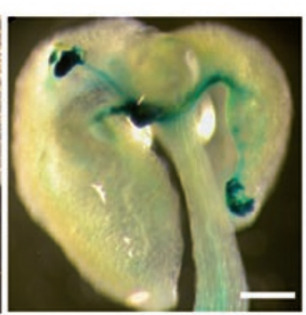

D CYCB1::GUS
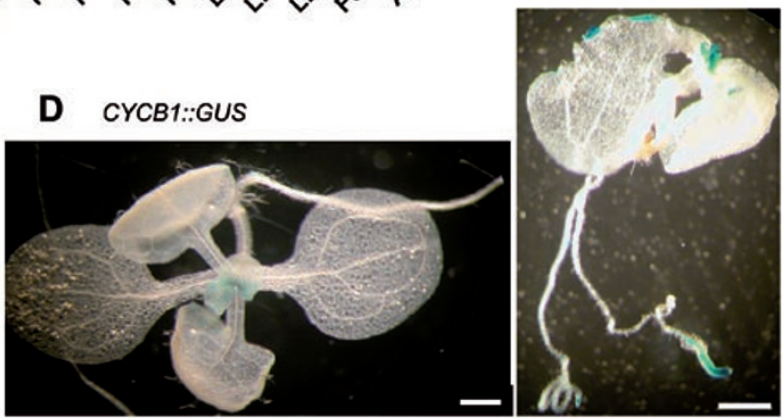

Figure 3 Expression analysis of embryonic and stem cell regulatory genes in Atring1a Atring1b. (A) Quantitative RT-PCR analysis of gene expression in 2-week-old seedlings. Relative expression levels are shown as induction fold in Atring1a Atring $1 b$ compared with wild-type (set as 1). Error bars represent standard deviation from triplicate repeats. (B) Spatial expression of the embryonic regulatory gene reporter AB/3::GUS in wild-type (upper panel) and Atring1a Atring1b (lower panel) plants. (C) Spatial expression of the stem cell regulatory gene reporter BP::GUS in wild-type (left panel) and Atring1a Atring1b (right panel) plants. (D) Spatial expression of the cell division reporter gene CYCB1::GUS in wild-type (left panel) and Atring1a Atring $1 \mathrm{~b}$ (right panel) plants. Scale bar $=1 \mathrm{~mm}$. 
in SAM and young leaves, in junction between shoot and root, in lateral root primordium and the root tip (left panel in Figure 3D). In Atringla Atringlb seedling, GUS activity is additionally visible in some regions in coty- ledons and leaves, and in pickle-root vasculature (right panel in Figure 3D). Together, the observed reporter gene expression patterns suggest that somatic embryogenesis and stem cell activities are associated with derepression
A

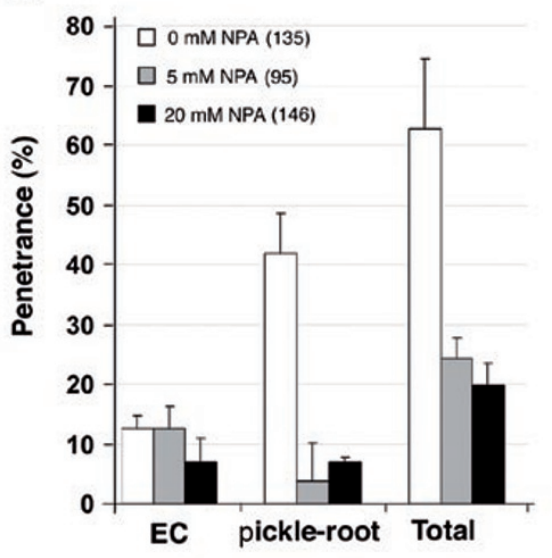

B

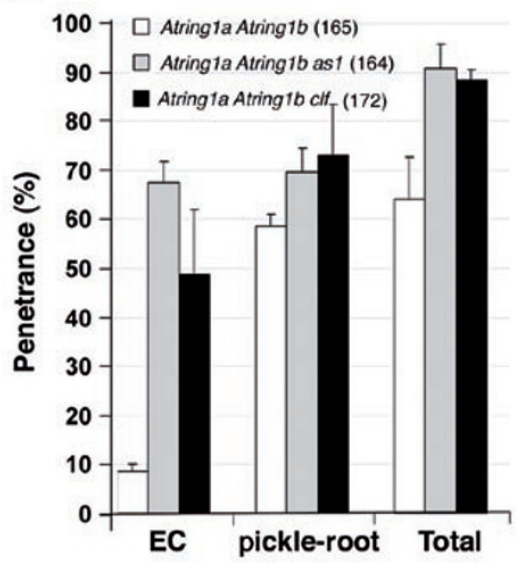

C

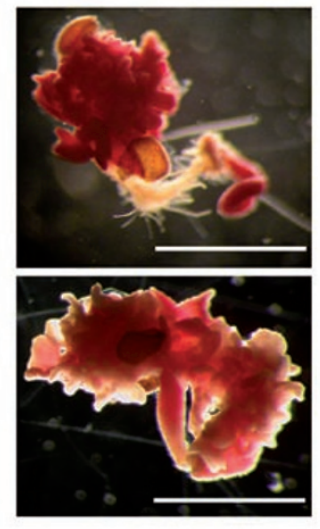

D

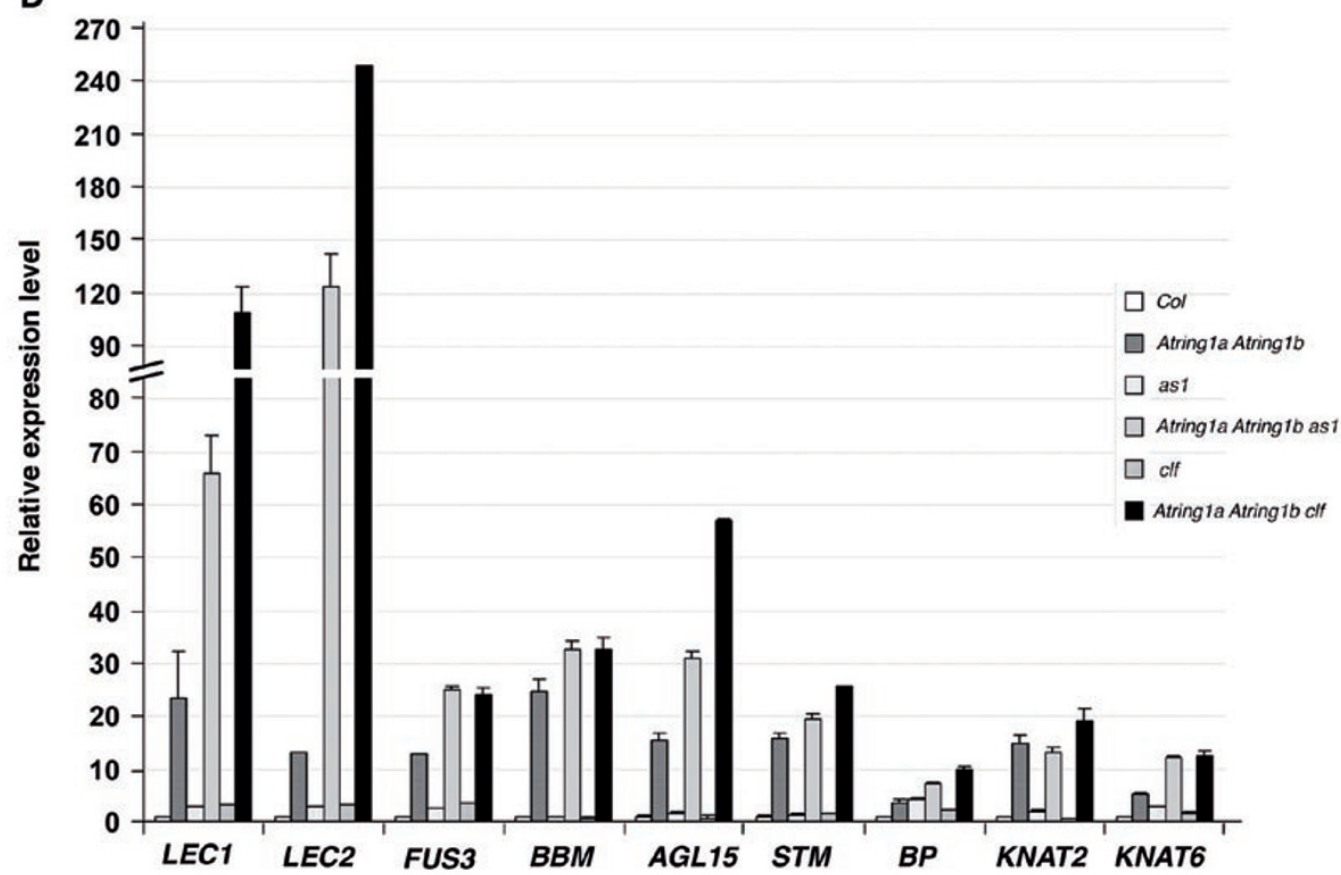

Figure 4 The auxin transporter inhibitor NPA inhibits and clf or as 1 enhances Atring1a Atring1b-mutant phenotypes. (A) Effect of NPA on the penetrance of embryonic calli (EC), pickle-root and total phenotypes in 1-month-old Atring1a Atring1b mutants. The number of scored plants is indicated for each growth condition within brackets. Error bars represent standard deviation from triplicate repeats. (B) Comparison of the penetrance of EC, pickle-root and total phenotypes in 1-month-old plants of the Atring1a Atring1b. Atring1a Atring1b clf and Atring1a Atring1b as 1 mutants. The number of scored plants is indicated for each mutant within brackets. Error bars represent standard deviation from triplicate repeats. (C) Phenotype of 1-month-old Atring1a Atring1b clf (upper panel) and Atring1a Atring1b as 1 (lower panel) plants stained by Fat Red. Scale bar $=1 \mathrm{~mm}$. (D) Quantitative RT-PCR analysis of gene expression in 1-month-old plants of the Atring1a Atring1b, Atring1a Atring1b clf and Atring1a Atring $1 \mathrm{~b}$ as 1 mutants. Relative expression levels are shown as induction fold in the mutant compared with wild-type (set as 1). Error bars represent standard deviation from triplicate repeats. 
of regulatory genes in the Atringla Atringlb mutant.

Derepression of embryonic traits of the Atringla Atringlb mutant is inhibited by NPA treatment, but enhanced by clf or as 1 mutation

To investigate whether auxin distribution plays a role in the establishment of the Atringla Atring $1 b$ mutant phenotype, we grew plants on culture medium in the presence of the auxin transport inhibitor 1-naphthylphthalamic acid (NPA). As shown in Figure 4A, NPA effectively reduced both EC and pickle-root penetrance in the Atringla Atring $1 b$ mutant. This is consistent with the well-established importance of the auxin gradient in embryogenesis and somatic embryo formation [23], and further indicates that a similar auxin function is necessary for expression of ectopic stem cell fate and embryonic traits in the Atringla Atringlb mutant.

$P K L$ and its homologue PKR2 have been shown to promote $\mathrm{H} 3 \mathrm{~K} 27 \mathrm{me} 3$ and activate the PRC2 genes $C L F$, $S W N$ and EMF2 [11, 27]. Consistently, the clf swn and emf 2 vrn 2 mutants also exhibit ectopic cell dedifferentiation and somatic embryogenesis [28]. Investigation of the Atringla Atringlb clf triple mutant [7] revealed that the EC and pickle-root penetrance of Atringla Atring $1 b$ were greatly and slightly enhanced by $c l f$, respectively (Figure 4B and 4C). ASYMMETRIC LEAVES1 (AS1), encoding a MYB-domain transcription factor, is involved in repression of the Class I KNOX genes $B P, K N A T 2$ and KNAT6 to promote leaf formation [29], and $p k l$ has been shown to enhance as 1 effects [30]. To examine the genetic interaction between as 1 and Atringla Atringlb, we obtained and studied the Atring 1 a Atring 1 b as 1 triple mutant. We found that as 1 greatly enhanced EC phenotype and frequency, but only slightly the pickle-root penetrance, in the Atringla Atring1b mutant (Figure 4B and 4C). Consistent with their enhanced phenotype, both the Atringla Atringlb clf and Atringla Atringlb as 1 triple mutants showed elevated derepression of embryonic and stem cell regulatory genes (Figure 4D). Taken together, these genetic data suggest that several complexes in conjunction with PRC2 and PRC1-like components act in repression of embryonic and stem cell regulatory genes to prevent dedifferentiation of somatic cells.

Identification of BMI1 homologues as putative PRC1-like components

To investigate the Arabidopsis BMI1-homologues as potential PRC1-like components, we first examined their physical interaction with LHP1, AtRING1a and AtRING1b in yeast two-hybrid assays. We found that AtBMI1a, AtBMI1b and AtBMI1c could bind with AtRING1a or AtRING1b, but only AtBMI1c could bind with LHP1 (Figure 5A). Consistent with previous reports [7, 31], we found that AtRING1a binds LHP1 and that LHP1 binds to itself (Figure 5A). To further confirm protein-protein interactions, we produced glutathione $S$ transferase (GST) fusion proteins, GST-AtBMI1a, GSTAtBMI1b and GST-AtBMI1c, and used them in pulldown assays. The assays were performed on total protein extract prepared from transgenic plants expressing FLAGAtRING1a [7]. As shown in Figure 5B, the anti-FLAG antibody detected FLAG-AtRING1a in input as well as in pulldown fractions by GST-AtBMI1a, GST-AtBMI1b or GST-AtBMI1c, but not by GST alone. Similarly, our previous study showed that GST-LHP1 can pulldown FLAG-AtRING1a [7]. Taken together, these observed physical interactions support that AtBMI, AtRING1 and LHP1 could form protein complexes.

Although all three proteins AtBMI1a, AtBMI1b and AtBMI1c show sequence homologies with mammalian BMI1 (Supplementary information, Figure S1), they are differently related. AtBMI1a and AtBMI1b amino acid sequences show $68 \%$ identity and $80 \%$ similarity to each other, but only show, respectively, $44 \%$ and $50 \%$ identity with AtBMI1c. It was previously reported that simultaneous loss-of-function of AtBMII a/DRIP1 and AtBMIIb/ $D R I P 2$ delays plant development and growth [9]. We obtained the corresponding T-DNA insertion mutants, Atbmila (previously named drip1-1 in Qin et al. [9]) and Atbmilb (previously named drip2-1 in Qin et al. [9]), and we generated the double mutant Atbmila Atbmilb. In support of a PRC1-like function for AtBMIla and AtBMIlb, we found that the Atbmila Atbmilb double mutant (but not the single mutants) showed EC and pickleroot phenotypes (Figure 5C-E and Supplementary information, Figure S2), which are largely similar to those of the Atringla Atring $1 b$ mutant. The penetrance of EC and pickle-root was nearly $18 \%$ and $8 \%$, respectively, and together account for $\sim 21 \%$ of Atbmila Atbmilb-mutant plants showing derepression of embryonic traits (Figure 5F). Consistent with derepression of embryonic traits, upregulation of expression in Atbmila Atbmilb was detected to varying extents for $C U C 1, W O X 5, W O X 8$, $A G L 15$, PIN2, BBM, LEC1, LEC2, FUS3 and ABI3 (Figure $5 \mathrm{G})$.

Taken together, our data suggest that AtRING1 and AtBMI1 proteins play non-redundant functions within a common PRC1-like complex involved in repression of regulatory gene transcription and embryonic traits during post-embryonic plant growth.

Gene expression analysis reveals a reciprocal repression between AtRING1 and AtBMII genes

The presence of multiple genes encoding ring-finger 

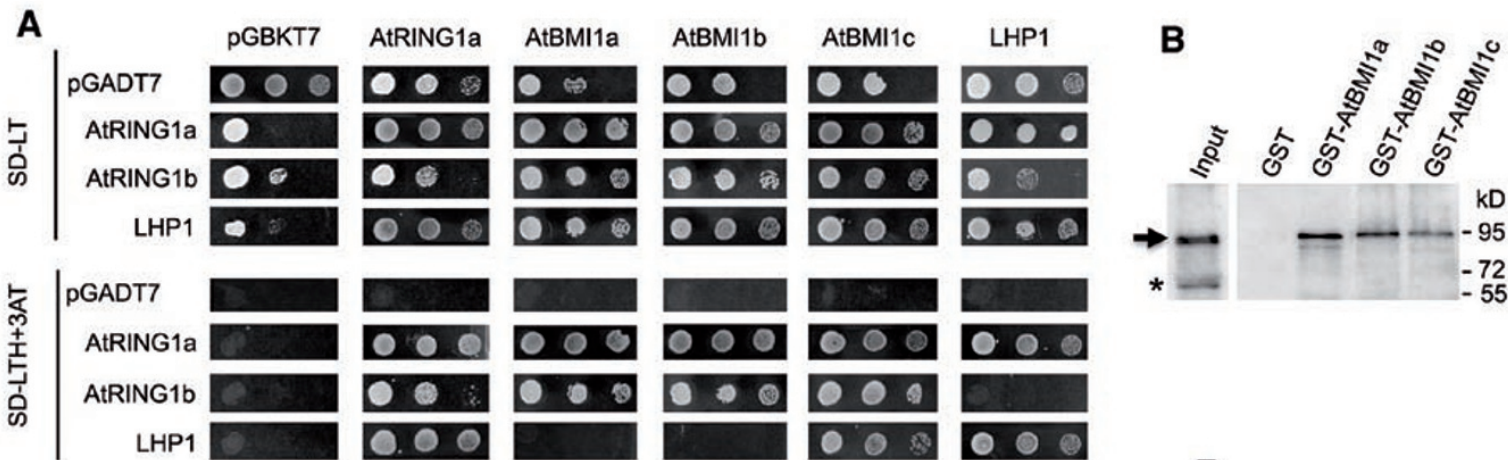

\section{$\mathbf{F}$}
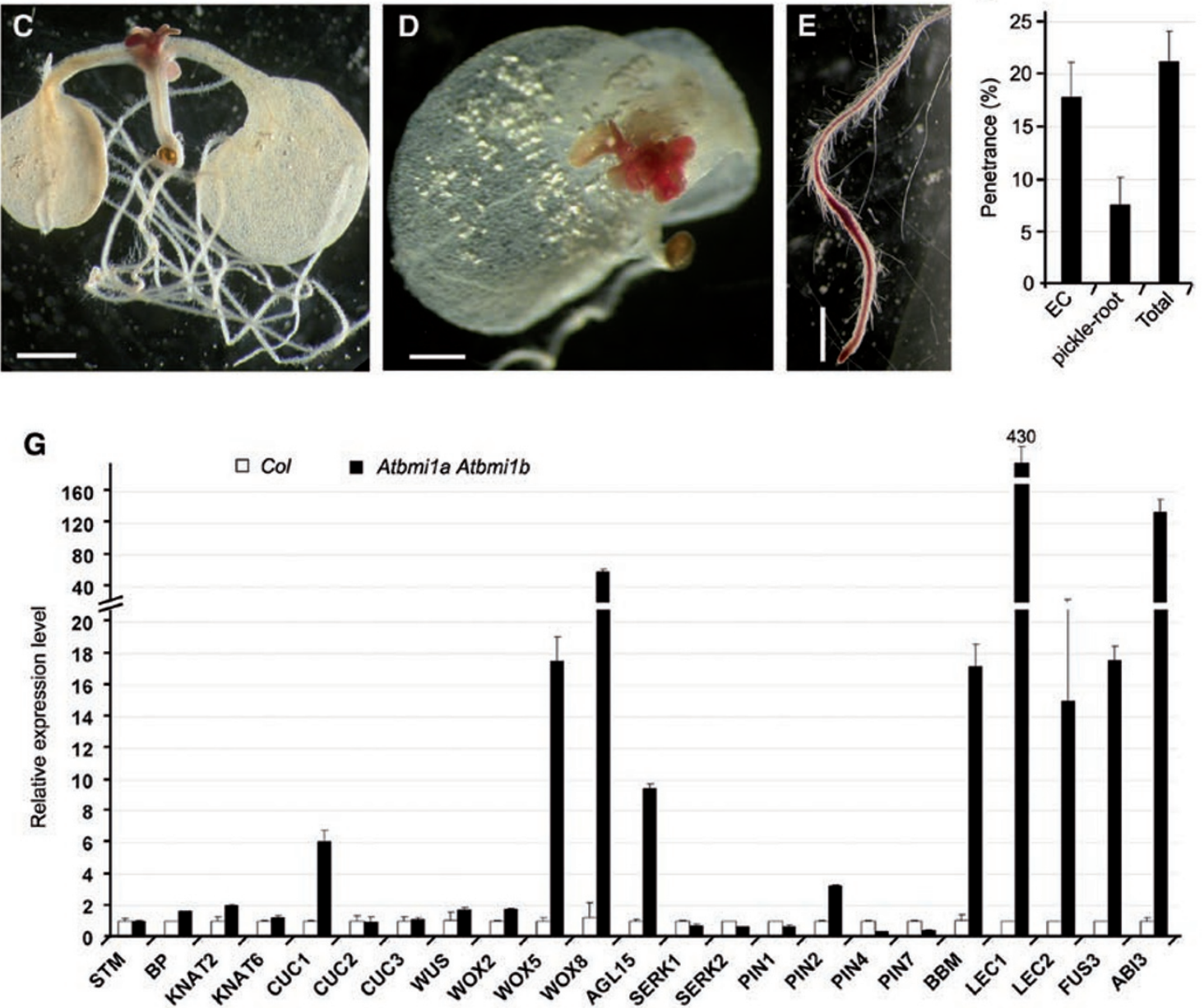

Figure 5 Examination of Arabidopsis BMI1 homologs as putative PRC1-like components. (A) Yeast two-hybrid assay for protein-protein interaction between putative PRC1-like components. Dilution $(\times 10)$ series of yeast cells coexpressing the indicated proteins from the pGBKT7 and pGADT7 vectors were plated onto SD-LT or selective medium SD-LTH+3AT. Growth of yeast cells on SD-LTH+3AT plate indicates positive interaction. (B) Pull-down assay. Total protein extract from 15-day-old seedlings expressing FLAG-AtRING1a was subdivided into four and incubated with GST-AtBMI1a-, GST-AtBMI1b-, GSTAtBMI1c- or GST-coated beads. The pull-down fractions were analyzed by western blotting with antibodies against the FLAG epitope. Arrowhead indicates band corresponding to the size of FLAG-AtRING1a. Asterisk indicates band of an unknown protein, present in input but absent from pull-down fractions. (C-E) Similar to the Atring1a Atring1b mutant, the Atbmi1a Atbmi1b mutant shows Fat Red staining in arrested SAM (C), in somatic embryonic calli (D) and in pickle-root regions (E). Scale bar $=1 \mathrm{~mm}$. (F) Penetrance of embryonic calli (EC), pickle-root and total phenotypes in 1-month-old Atbmi1a Atbmi1b plants. A sum of 263 plants was scored. Error bars represent standard deviation from triplicate repeats. (G) Quantitative RT-PCR analysis of gene expression in 2-week-old seedlings. Relative expression levels are shown as induction fold in Atbmi1a Atbmi1b compared with wild-type (set as 1). Error bars represent standard deviation from triplicate repeats. 
components of the PRC1-like complex is intriguing. To gain insight into their regulation, we investigated expression of AtRING1a, AtRING1b, AtBMIIa, AtBMIlb, $A t B M I 1 c$ and several others genes involved in repression of embryonic traits and stem cell activity in somatic cells. We found that $A t B M I I c$ and, to a less degree, $A t B$ $M I l a$ and $A t B M I I b$ were all upregulated in the Atringla Atring $1 b$ mutant (Figure 6A), and that AtRINGla and AtRING1b, as well as AtBMI1c, were upregulated in the Atbmila Atbmilb mutant (Figure 6B). PKR2 expression was also increased in the Atringla Atringlb mutant and to a less degree in the Atbmila Atbmilb mutant. Slightly decreased $P K L$ expression was observed in the Atbmila Atbmilb mutant and an increase of VAL1 expression was observed in the Atringla Atring $1 b$ mutant. The remaining genes examined, including $L H P 1, C L F, S W N, E M F 2$ and $V R N 2$, did not show expression changes in either double mutant (Figure 6). The observation of reciprocal repression of AtRING1a/AtRING1b and AtBMIIa/AtB$M I 1 b$ suggests that these PRC1-like ring-finger genes are themselves repressed by the PRC1-like complex.

\section{Discussion}

\section{PRC1-like complexes in Arabidopsis}

Currently, several lines of evidence support the presence of PRC1-like complexes in Arabidopsis. First, LHP1 binds H3K27me3 through its chromodomain and this binding is crucial for LHP1 function [5, 6, 32], supporting the hypothesis that LHP1 is functionally analo- gous to the animal PRC1 component Pc in Arabidopsis. Second, AtRING1a and AtRING1b, as well as AtBMIla, AtBMI1b and AtBMI1c, show high sequence homology and similar domain organization to their animal homologues RING1 and BMI1 [7, 8]; both RING1 and BMI1 are core components of the PRC1 complex in animals. Third, physical interaction has been detected for LHP1 with AtRING1a or AtBMI1c; for AtRING1a with AtRING1b, AtBMI1a, AtBMI1b or AtBMI1c; and for AtRING1b with AtBMI1a, AtBMI1b or AtBMI1c (Xu and Shen [7]; this study), supporting several possible combinations of multiprotein complexes. In another line of supporting evidence, GUS reporter expression analysis reveals that AtRING1a (this study), AtBMIIa/DRIP1 [9] and LHP1 [33] have similar expression patterns, with high expression levels found in plant tissues containing actively proliferating cells, supporting possible ensemble action of these genes. Finally, AtRING1a and AtRING1b share with LHP1 a common mechanism in repression of some PcG-target genes, which does not involve any change in $\mathrm{H} 3 \mathrm{~K} 27 \mathrm{me} 3$ deposition [7] and is consistent with the known PRC1 function in stabilization of gene repression downstream of PRC2-mediated H3K27me3 deposition.

Among the five ring-finger genes, AtRING1 $a$ and AtRING $1 b$ show a redundant function, and AtBMIla and $A t B M I 1 b$ are also functionally redundant. Consistent with the view that AtRING1 and AtBMI1 play non-redundant roles within a PRC1-like complex, the Atringla Atringlb and Atbmila Atbmilb mutants show
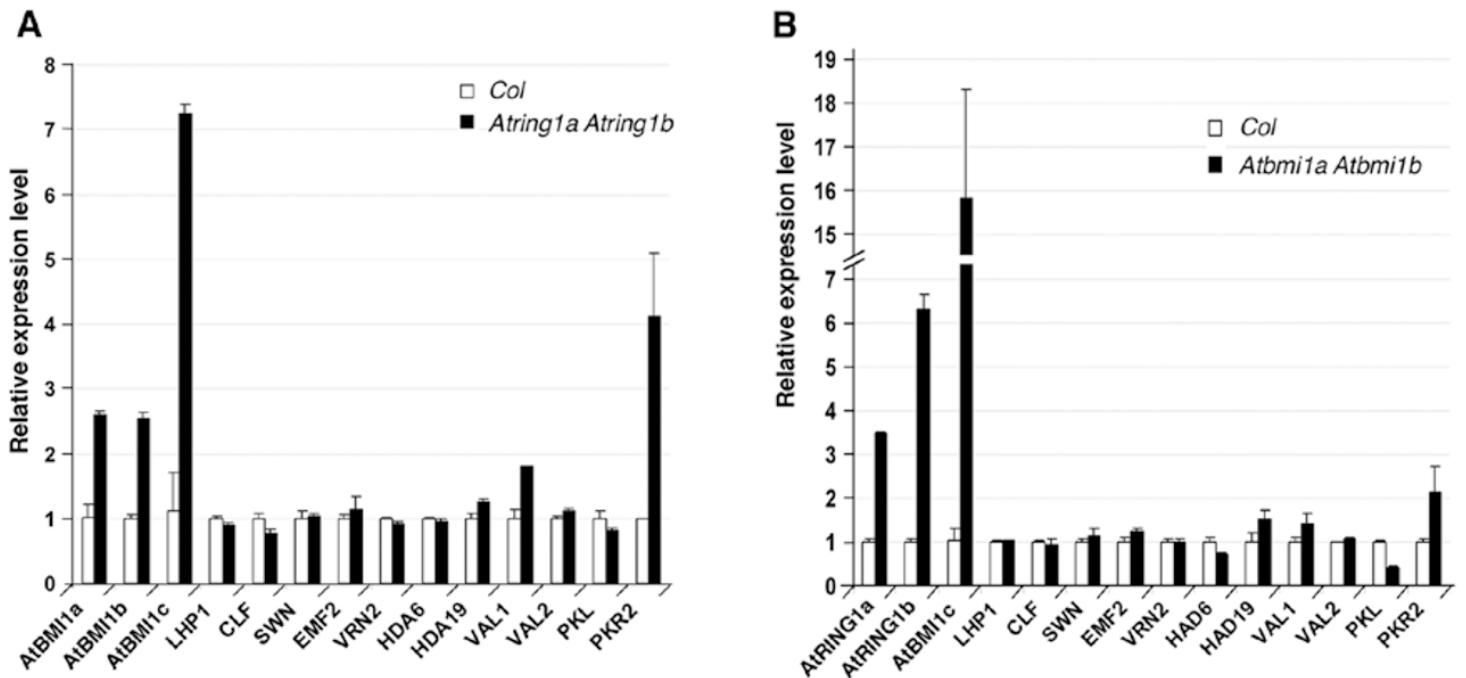

Figure 6 Quantitative RT-PCR analysis of gene expression in 2-week-old seedlings of the Atring1a Atring1b (A) and Atbmi1a Atbmi1b (B) mutants. Relative expression levels are shown as induction fold in the mutant compared with wild-type (set as 1). Error bars represent standard deviation from triplicate repeats. 
largely similar embryonic derepressive phenotypes. So far, the function of AtBMIIC has not yet been examined. Interestingly, AtBMIIC expression is upregulated in both the Atringla Atringlb and Atbmila Atbmilb mutants, AtRING1 $a$ and AtRING1b expression is upregulated in the Atbmila Atbmilb mutant and AtBMIIa and AtBMIIb expression is upregulated in the Atringla Atring $1 b \mathrm{mu}-$ tant; thus, revealing that repression of the PRC1-like ring-finger genes is regulated by themselves. Distinct from the largely similar phenotypes observed between the Atringla Atring1b and Atbmila Atbmilb mutants, the $\operatorname{lhp} 1$ mutant has a relatively different phenotype [31], and $l h p l$ enhances the Atringla Atringlb phenotype [7]. This indicates that LHP1 might not be the only Arabidopsis protein recognizing $\mathrm{H} 3 \mathrm{~K} 27 \mathrm{me} 3$, but that there are other LHP1-independent pathways acting in parallel for AtRING1 and AtBMI1 function. Future identification of novel components and further biochemical characterization of PRC1-like complexes will provide further insight into PcG-mediated gene silencing in plants.

$P R C 2$ and PRC1-like complexes in repression of embryonic traits and stem cell activities in somatic cells

The Atringla Atring1b and Atbmila Atbmilb (drip1-1 drip2-1) mutants show pleiotropic phenotypes [7,9]. Our study focusing on these mutants during seedling growth reveals a crucial function of PRC1-like complexes in repression of embryogenesis and stem cell activities for proper vegetative growth. Plants displaying a severe mutant phenotype fail to form true leaves and display EC and/or pickle-root phenotypes, which could occur simultaneously within the same plant. EC formation could be observed on various regions of the plant, including the SAM region, cotyledons, leaves and roots, suggesting a general rather than organ-specific requirement of AtRING1a and AtRING1b, as well as AtBMI1a and AtBMI1b in embryonic trait repression. Pleiotropy and variable expressivity of mutant phenotypes among individual plants are not specific characteristics of Atringla Atringlb and Atbmila Atbmilb, but occur frequently in many chromatin modification or remodeling-defective mutants in plants. This variability could be associated with the high degree of plasticity of plant growth and development, which is essential for plants to cope with environmental changes. While the penetrance of EC is similar in the Atringla Atringlb and Atbmila Atbmilb mutants (17$18 \%$ ), the penetrance of pickle-root phenotype in Atringla Atringlb $(\sim 50 \%)$ is significantly higher than that in Atbmila Atbmilb ( $8 \%)$. It will be interesting in future experiments to investigate whether $A t B M I 1 c$ has a redundant function with AtBMII $a$ and AtBMIIb in repression of embryonic traits in roots. The penetrance of pickle- root phenotype was previously reported to be low in $\mathrm{pkl}$ (< $10 \%)$ but enhanced in $p k l p k r 2$ (32-40\%, Aichinger et al. [11]).

Consistent with the strongly derepressed embryonic traits, many regulatory genes involved in embryogenesis and stem cell maintenance are upregulated in the Atringla Atring $1 b$ mutant. These include the key embryonic regulatory genes $L E C 1, L E C 2, F U S 3, A B I 3$ and $B B M$ [18-22], the embryonic competence-enhanced gene $A G L 15$ [16], the key RAM-regulatory and basal embryoaxis cell fate genes $W O X 5$ and $W O X 8$ [15], the key SAM-regulatory genes STM, BP, KNAT2 and KNAT6 [7], the organ boundary regulatory genes $C U C 1, C U C 2$ and $C U C 3$ [13] and the auxin transporter genes PIN1 and PIN2 [24]. Remarkably, treatment with the auxin transporter inhibitor NPA can reduce the penetrance of embryonic traits in Atringla Atringlb, indicating that the normal requirement of polar auxin gradient in embryogenesis [23] is maintained in ectopic embryonic trait development in the mutant. The $L E C 1, L E C 2, F U S 3, A B I 3$, $B B M, A G L 15, W O X 5, W O X 8, C U C 1$ and PIN2 genes were also found to be upregulated to varying extents in the Atbmila Atbmilb mutant. Interestingly, the Class I KNOX genes (STM, BP, KNAT2 and KNAT6) were upregulated in Atringla Atring $1 b$, but barely changed in Atbmila Atbmilb. This is consistent with the highly fasciated stem phenotype observed in Atringla Atring $1 b$ [7] but not in Atbmila Atbmilb (drip1-1 drip2-1, Qin et al. [9]). Again, the difference between Atringla Atring $1 b$ and Atbmila Atbmilb might be explained by the existing AtBMIIc function. Alternatively, as AtBMIIa/DRIPI and $A t B M I 1 b / D R I P 2$ were shown to target the transcription factor DREB2A to proteasome degradation [9], the AtRING1 and AtBMI1 proteins might have additionally independent roles in modifying transcription factors to modulate gene transcription.

The LEC1, LEC2, FUS3 and ABI3 genes were also upregulated in $p k l$ and more drastically in $p k l p k r 2$ [11, 27]. The chromodomain/helicase/DNA-binding domain (CHD3) proteins PKL and PKR2 can activate several PRC2 component genes including $C L F, S W N$ and $E M F 2$, thus suggesting that derepression of embryonic traits and regulatory genes in $p k l$ and $p k l p k r 2$ is caused by reduced PRC2 activity [11, 27]. Distinctively, the derepression of embryonic traits and regulatory genes in Atring $1 a$ Atring $1 b$ and Atbmila Atbmilb is not associated with any detectable changes in expression of the PRC2 component genes. Upregulation of $P K R 2$ was detected in both Atring $1 a$ Atring $1 b$ and Atbmila Atbmilb, suggesting a feedback loop that could compensate reduced PRC1-like activity. Nevertheless, effectiveness of such a regulatory mechanism will need to be verified in future experiments. 
Both clf swn and emf2 vrn 2 show ectopic formation of embryo-like structures [28], and at least in the case of clf swn the structures developing from above-ground organs exhibit Fat Red staining [11]. Consistently, ectopic derepression of LEC1, FUS3 and STM was observed in clf swn $[34,35]$. Our triple mutant analysis revealed that clf enhances the Atringla Atringlb-mutant phenotype and the derepression of LEC1, FUS3 and STM, as well as LEC2, AGL15, BP and KNAT6 (Figure 4). We also found that as 1 has a similar function in enhancing the Atringla Atringlb-mutant phenotype and the Atringla Atring $1 b$ as 1 triple mutant exhibits elevated derepression of embryonic and stem cell regulatory genes (Figure 4), suggesting that AS1 establishes leaf cell fate via a link with the PcG pathway. A previous study showed that the as 1 -mutant phenotype is enhanced by $p k l$, which is associated with elevated derepression of BP and KNAT2 [30]. Further experiments are necessary to better understand the molecular mechanisms underlying the link between AS1 and the PKL and PcG pathways.

In conclusion, this work together with previous studies, establishes the crucial function of PcG-mediated gene silencing mechanisms in the maintenance of stable repression of embryonic traits for post-embryonic plant growth and development. The strong somatic embryogenesis phenotypes associated with derepression of a large number of embryonic and stem cell regulatory genes, as observed in Atringla Atringlb and in Atbmila Atbmilb (drip1-1 drip2-1), reveal a central role of the PRC1like ring-finger components, which is in agreement with the well-established function of animal PRC1, known to act downstream of PRC2 in maintenance (as opposed to initiation) of PcG-mediated gene silencing. Several pathways, including PRC2, PKL and AS1, likely work in conjunction with the PRC1-like complexes in stable repression of the embryonic program in somatic cells. Such a stable yet flexible repression system might be advantageous to cope with the highly frequent cell differentiation occurring during plant organogenesis and with the remarkable developmental plasticity of plants.

\section{Materials and Methods}

\section{Plant materials and growth conditions}

All Arabidopsis thaliana mutants were derived from the Columbia ecotype. Mutants as 1-1, Atbmila/drip1-1 (WiscDsLox437G06) and Atbmilb/drip2-1 (SALK_145041) were obtained from the Arabidopsis Biological Resource Center (ABRC, http://www.Arabidopsis.org). The Atringla Atringlb double mutant and the Atringla Atringlb clf triple mutant have been previously described [7]. Other combined mutant and reporter gene lines were obtained by appropriate genetic crosses. For plant growth, seeds were surface sterilized ( $70 \%$ and $95 \%$ ethanol for 10 min) and plated on MS medium (MS salts, $1 \%$ sucrose (pH 5.8), $0.9 \%$ bactoagar). For NPA effect assay, the MS medium was supplemented with NPA (Sigma-Aldrich, http://www.sigmaaldrich. com) at the specified concentrations. After stratification at $4{ }^{\circ} \mathrm{C}$ for 2 days in the dark, plates were incubated in a growth chamber at $22{ }^{\circ} \mathrm{C}$ under a 16 -h light/8-h dark photoperiod.

\section{Plasmid construction and plant transformation}

For AtRING1a::AtRING1a-GUS construction, the genomic DNA fragment containing the upstream promoter and the entire coding region of the AtRINGla gene (from -1359 bp to +4356 bp) was PCR-amplified from BAC K9L2 using specific primers (Supplementary information, Table S1). The DNA fragment was digested and cloned into pBI101 (Clontech, http://www.clontech. com) using SalI and BamHI. The GUS reporter gene was cloned in frame at the C-terminal end of AtRINGla (AtRINGla::AtRINGlaGUS). The binary vector was introduced into Agrobacterium tumefaciens GV3101, which was used to transform Arabidopsis plants by the floral-dip method [36].

\section{GUS histochemical assays}

For histochemical GUS activity assays, seedlings were submerged in $90 \%$ acetone for $30 \mathrm{~min}$ on ice, washed twice with $50 \mathrm{mM}$ sodium phosphate buffer $(\mathrm{pH} 7.2)$ for $15 \mathrm{~min}$ at room temperature and subsequently incubated in staining solution $(0.1$ $\mathrm{M}$ sodium phosphate buffer ( $\mathrm{pH} 7.2), 0.5 \mathrm{mM} \mathrm{Fe}(\mathrm{CN})_{2}, 0.5 \mathrm{mM}$ $\mathrm{Fe}(\mathrm{CN})_{3}, 0.1 \%$ Tween-20 and $2 \mathrm{mM}$ 5-bromo-4-chloro-3-indolyl$\beta$-D-glucuronide) at $37^{\circ} \mathrm{C}$ for $1-12 \mathrm{~h}$, depending on the activity of each reporter gene construct. Seedlings were cleared by incubation overnight in $70 \%$ ethanol at $4{ }^{\circ} \mathrm{C}$.

\section{Fat Red staining}

Whole seedlings were stained overnight with a filtered solution of Fat Red 7B (Sigma-Aldrich, http://www.sigmaaldrich.com), as previously described [11]. For quantitative penetrance analysis, plants were grown and analyzed in triplicate from three plates for each experiment, and the experiment was repeated independently at least two times.

\section{Starch staining}

Roots from 1-month-old plants were cleared with chloral hydrate solution ( $8 \mathrm{~g}$ chloral hydrate, $2 \mathrm{ml}$ glycerol, $1 \mathrm{ml} \mathrm{H}_{2} \mathrm{O}$ ) and then stained with Lugol's solution $\left(5 \mathrm{~g} \mathrm{I}_{2}, 10 \mathrm{~g} \mathrm{KI}, 85 \mathrm{ml} \mathrm{H}_{2} \mathrm{O}\right)$.

\section{$R N A$ isolation and quantitative RT-PCR analysis}

Total RNA was isolated using the NucleoSpin RNA Plant kit (Macherey-Nagel, http://www.mn-net.com). Quantitative RT-PCR was performed on a light cycler $480 \mathrm{II}$ (Roche), according to the manufacturer's instructions. Reaction volumes were scaled to 10 $\mu 1$ final volume and were comprised of $5 \mu$ of SYBR Green PCR master mix (Roche, http://www.roche-applied-science.com), $2 \mu 1$ of primer mix and $1 \mu \mathrm{l}$ of template cDNA. Each sample was analyzed in triplicate, and $P P 2 A, E X P$ and Tip41 were used as internal reference genes.

\section{Yeast two-hybrid assay}

The entire ORFs of the AtBMIIa, AtBMIIb and AtBMIIc cDNAs were amplified using gene-specific primers and subsequently cloned into the pGBKT7 and pGADT7 vectors (Clontech, http:// 
www.clontech.com). Vectors containing LHP1, AtRING1a and AtRINGIb have been previously described [7]. Bait and prey constructs were cotransformed into the yeast strain pJ69-4a, and transformants were selected by growth on a synthetic defined (SD) medium lacking Leu and Trp (SD-LT). The bait-prey interaction was tested by growth of the transformants on SD medium lacking Leu, Trp and His plus $6 \mathrm{mM}$ 3-amino-1,2,4-triazole (SD-LTH+3AT).

\section{Pull-down assay}

The AtBMIIa, AtBMIIb and AtBMIc cDNAs were cloned into the $B a m$ HI-XhoI sites of pGEX-4T-1 for production of GST-fused proteins. Production and purification of GST-AtBMI1a, GSTAtBMI1b and GST-AtBMI1c proteins were performed according to the manufacturer's recommendation (Amersham-Pharmacia Biotech, http://www.amersham.com). Pulldown experiments were performed as previously described [7].

\section{Acknowledgments}

We thank Lin Xu for generation of AtRING1a::AtRING1a-GUS transgenic plants and of Atringla Atringlb as 1 mutant, Francois Parcy (Laboratoire de physiologie cellulaire végétale, Grenoble) for providing the ABI3::GUS reporter line and Emily J McCallum for critical reading of this manuscript. AM is supported by a research-training fellowship from the Luxembourg Ministère de la Culture, de l'Enseignement Supérieur et de la Recherche. This work was funded by the French Centre National de la Recherche Scientifique (CNRS) and in part by the French Agence Nationale de la Recherche (ANR-08-BLAN-0200-CSD7).

\section{References}

1 Schuettengruber B, Chourrout D, Vervoort M, Leblanc B, Cavalli G. Genome regulation by polycomb and trithorax proteins. Cell 2007; 128:735-745.

2 Pien S, Grossniklaus U. Polycomb group and trithorax group proteins in Arabidopsis. Biochem Biophys Acta 2007; 1769:375-382.

3 Alvarez-Venegas R. Regulation by polycomb and trithorax group proteins in Arabidopsis. In: Chang $\mathrm{C}$ ed. The Arabidopsis Book. Rockville, MD: American Society of Plant Biologists, 2010.

4 Shen $\mathrm{WH}, \mathrm{Xu}$ L. Chromatin remodeling in stem cell maintenance in Arabidopsis thaliana. Mol Plant 2009; 2:600-609.

5 Turck F, Roudier F, Farrona S, et al. Arabidopsis TFL2/LHP1 specifically associates with genes marked by trimethylation of histone H3 lysine 27. PLoS Genet 2007; 3:e86.

6 Zhang X, Germann S, Blus BJ, Khorasanizadeh S, Gaudin V, Jacobsen SE. The Arabidopsis LHP1 protein colocalizes with histone H3 Lys27 trimethylation. Nat Struct Mol Biol 2007; 14:869-871.

$7 \mathrm{Xu}$ L, Shen WH. Polycomb silencing of KNOX genes confines shoot stem cell niches in Arabidopsis. Curr Biol 2008; 18:1966-1971.

8 Sanchez-Pulido L, Devos D, Sung ZR, Calonje M. RAWUL: a new ubiquitin-like domain in PRC1 ring finger proteins that unveils putative plant and worm PRC1 orthologs. BMC Genomics 2008; 9:308.
9 Qin F, Sakuma Y, Tran LS, et al. Arabidopsis DREB2A-interacting proteins function as RING E3 ligases and negatively regulate plant drought stress-responsive gene expression. Plant Cell 2008; 20:1693-1707.

10 Masubelele NH, Dewitte W, Menges M, et al. D-type cyclins activate division in the root apex to promote seed germination in Arabidopsis. Proc Natl Acad Sci USA 2005; 102:1569415699.

11 Aichinger E, Villar CB, Farrona S, Reyes JC, Hennig L, Kohler C. CHD3 proteins and polycomb group proteins antagonistically determine cell identity in Arabidopsis. PLoS Genet 2009; 5:e1000605.

12 Ogas J, Cheng JC, Sung ZR, Somerville C. Cellular differentiation regulated by gibberellin in the Arabidopsis thaliana pickle mutant. Science 1997; 277:91-94.

13 Vroemen CW, Mordhorst AP, Albrecht C, Kwaaitaal MA, de Vries SC. The CUP-SHAPED COTYLEDON3 gene is required for boundary and shoot meristem formation in Arabidopsis. Plant Cell 2003; 15:1563-1577.

14 Laux T, Mayer KF, Berger J, Jurgens G. The WUSCHEL gene is required for shoot and floral meristem integrity in Arabidopsis. Development 1996; 122:87-96.

15 Breuninger H, Rikirsch E, Hermann M, Ueda M, Laux T. Differential expression of $W O X$ genes mediates apical-basal axis formation in the Arabidopsis embryo. Dev Cell 2008; 14:867876.

16 Harding EW, Tang W, Nichols KW, Fernandez DE, Perry $\mathrm{SE}$. Expression and maintenance of embryogenic potential is enhanced through constitutive expression of AGAMOUS-Like 15. Plant Physiol 2003; 133:653-663.

17 Schmidt ED, Guzzo F, Toonen MA, de Vries SC. A leucinerich repeat containing receptor-like kinase marks somatic plant cells competent to form embryos. Development 1997; 124:2049-2062.

18 Boutilier K, Offringa R, Sharma VK, et al. Ectopic expression of $B A B Y B O O M$ triggers a conversion from vegetative to embryonic growth. Plant Cell 2002; 14:1737-1749.

19 Lotan T, Ohto M, Yee KM, et al. Arabidopsis LEAFY COTYLEDON1 is sufficient to induce embryo development in vegetative cells. Cell 1998; 93:1195-1205.

20 Giraudat J, Hauge BM, Valon C, Smalle J, Parcy F, Goodman HM. Isolation of the Arabidopsis ABI3 gene by positional cloning. Plant Cell 1992; 4:1251-1261.

21 Luerssen H, Kirik V, Herrmann P, Misera S. FUSCA3 encodes a protein with a conserved VP1/AB13-like B3 domain which is of functional importance for the regulation of seed maturation in Arabidopsis thaliana. Plant J 1998; 15:755-764.

22 Stone SL, Kwong LW, Yee KM, et al. LEAFY COTYLEDON2 encodes a B3 domain transcription factor that induces embryo development. Proc Natl Acad Sci USA 2001; 98:11806-11811.

23 Verdeil JL, Alemanno L, Niemenak N, Tranbarger TJ. Pluripotent versus totipotent plant stem cells: dependence versus autonomy? Trends Plant Sci 2007; 12:245-252.

24 Blilou I, Xu J, Wildwater M, et al. The PIN auxin efflux facilitator network controls growth and patterning in Arabidopsis roots. Nature 2005; 433:39-44.

25 To A, Valon C, Savino G, et al. A network of local and redundant gene regulation governs Arabidopsis seed maturation. Plant Cell 2006; 18:1642-1651. 
26 Colon-Carmona A, You R, Haimovitch-Gal T, Doerner P. Technical advance: spatio-temporal analysis of mitotic activity with a labile cyclin-GUS fusion protein. Plant J 1999; 20:503-508.

27 Zhang H, Rider SD Jr, Henderson JT, et al. The CHD3 remodeler PICKLE promotes trimethylation of histone $\mathrm{H} 3$ lysine 27. J Biol Chem 2008; 283:22637-22648.

28 Chanvivattana Y, Bishopp A, Schubert D, et al. Interaction of Polycomb-group proteins controlling flowering in Arabidopsis. Development 2004; 131:5263-5276.

29 Byrne ME, Barley R, Curtis M, et al. Asymmetric leaves1 mediates leaf patterning and stem cell function in Arabidopsis. Nature 2000; 408:967-971.

30 Ori N, Eshed Y, Chuck G, Bowman JL, Hake S. Mechanisms that control knox gene expression in the Arabidopsis shoot. Development 2000; 127:5523-5532.

31 Gaudin V, Libault M, Pouteau S, et al. Mutations in LIKE HETEROCHROMATIN PROTEIN 1 affect flowering time and plant architecture in Arabidopsis. Development 2001;
128:4847-4858.

32 Exner V, Aichinger E, Shu H, et al. The chromodomain of LIKE HETEROCHROMATIN PROTEIN 1 is essential for H3K27me3 binding and function during Arabidopsis development. PLoS ONE 2009; 4:e5335.

33 Kotake T, Takada S, Nakahigashi K, Ohto M, Goto K. Arabidopsis TERMINAL FLOWER 2 gene encodes a heterochromatin protein 1 homolog and represses both FLOWERING LOCUS $T$ to regulate flowering time and several floral homeotic genes. Plant Cell Physiol 2003; 44:555-564.

34 Makarevich G, Leroy O, Akinci U, et al. Different Polycomb group complexes regulate common target genes in Arabidopsis. EMBO Rep 2006; 7:947-952.

35 Schubert D, Primavesi L, Bishopp A, et al. Silencing by plant Polycomb-group genes requires dispersed trimethylation of histone $\mathrm{H} 3$ at lysine 27. EMBO J 2006; 25:4638-4649.

36 Clough SJ, Bent AF. Floral dip: a simplified method for Agrobacterium-mediated transformation of Arabidopsis thaliana. Plant J 1998; 16:735-743.

(Supplementary information is linked to the online version of the paper on the Cell Research website.) 